\title{
Magnetic Resonance Venography Findings In A Group Of Patients With Multiple Sclerosis
}

\author{
Ali Qasim Al-Baghdady*, Nael Husain**, Samer Mohammed Saeed Ridha *, Zaki Noah Hasan***
}

\begin{abstract}
Background: Multiple sclerosis is a chronic heterogeneous demyelinating axonal and inflammatory disease involving the Central Nervous System [CNS] white matter with a possibility of gray matter involvement in which the insulating covers of nerve cells in the brain and spinal cord are damaged. This damage disrupts the ability of parts of the nervous system to communicate, resulting in a wide range of signs and symptoms. Cerebral venous insufficiency theory was raised as a possible etiology for the disease at 2008 by Zamboni an Italian cardiothoracic surgeon. This theory was defeated by Multiple Sclerosis[ MS] researchers and scientists who thought that the disease is an autoimmune rather than vascular.

Objective: To assess the findings of Magnetic Resonance Venography [MRV] in a group of patients with MS and to compare these results with a healthy control group Magnetic Resonance Venography[ MRV] findings.

Type of the study: A case - control cross sectional study. Methods: it was conducted at Neurosciences Hospital MS clinic from Oct.2014 to Dec. 2015, recruiting sequentially 50 patients who attend the MS clinic; 19 male and 31 females, their age was ranged from 16 to 53 year old. Diagnosis of MS was based on fulfillment of McDonald 2010 criteria for MS diagnosis, detailed history, examination and Expanded Disability Status Scale( EDSS)[see Appendix1] were recorded according to structured questionnaire forum. Each
\end{abstract}

patient was send for Magnetic Resonance Venography[ MRV] using 3 Tessla Phillips model Achieva, 2012. The results of MRV were interpreted by specialist radiologist. Statistical analyses were done using SPSS version 21 with $P$ value less than 0.05 was considered significant.

Results: Fifty patients with Multiple Sclerosis were involved in the study female 31 , male 19 , their ages were ranged between 21 and 60 year old. Magnetic Resonance Venography [MRV] findings suggest of venous thrombosis were seen in [4 / 50] $8 \%$ of patients and [46/50] $92 \%$ of the patients in this study have no findings of venous thrombosis. The comparison with the control group showed no significant statistical difference.

Conclusion: Magnetic Resonance Venography[MRV] finding is not statistically different between healthy people and Multiple Sclerosis patient.

Keywords: Multiple Sclerosis , Magnetic Resonance Venography, Nervous.

\section{Al-Kindy College Medical Journal 2017: Vol.13 No.1} Page: 116-121

\author{
* Neuro Science Hospital \\ **Mesan Al-Sader Teaching Hospital \\ ${ }^{* * *}$ Al-Kindy College of Medicine \\ Received $15^{\text {th }}$ Feb 2017, accepted in final $30^{\text {th }}$ March 2017 \\ Corresponding to : Ali Qasim Al-Baghdady
}

$\mathrm{M}$ ultiple sclerosis (MS) is the most common cause of non-traumatic disability in young adults. It is a chronic inflammatory demyelinating disease of the central nervous system (CNS) (1) .It's a demyelinating disease in which the insulating covers of nerve cells in the brain and spinal cord are damaged. This damage disrupts the ability of parts of the nervous system to communicate, resulting in a wide range of signs and symptoms, including physical, mental (2), and sometimes psychiatric problems (3). MS takes several forms, with new symptoms either occur in isolated attacks (relapsing forms) or progress over time (progressive forms). Symptoms may resolve completely between attacks ; however, a permanent neurological deficit occurs, especially as the disease advances and progress(4). A controversial hypothesis proposes a vascular rather than an immunologic cause for some cases of MS. In 2008, Paolo Zamboni described an association between MS and chronic cerebrospinal venous insufficiency (CCSVI)(5). The CCSVI hypothesis posits that stenosis of the main extracranial venous outflow pathways results in compromised drainage and a high rate of cerebral venous reflux. The CCSVI hypothesis has been linked with the potential effects of iron deposition in the brain parenchyma, which some authors suggest is modestly to strongly predictive of disability progression, lesion volume accumulation, and atrophy in some patients with MS $(6,7)$.

The aim of the study was To assess the findings of Magnetic Resonance Venography [MRV] findings in a group of patients with Multiple Sclerosis [MS] and to compare these results with a control group MRV findings.

Methods:A cross-sectional case control study of Magnetic Resonance Venography( MRV) finding in multiple sclerosis (MS) cases started from October 2014 to the December 2015 in which a thorough work up of 50 cases had MS was done. The patients were taken from (Neurosciences Hospital - MS Clinic -Baghdad / Iraq). The study recruited two groups of patients. The first group includes Multiple sclerosis patients who meet The MS clinic in Neurosciences Hospital. The second group 
was healthy control group selected from hospital staff. The patients were of both sexes. (19 male, 31 female), and age ranging from (19 years to 55 years). The MS group Inclusion Criteria were Patient should have fulfilling McDonald 2010 criteria of MS and having Normal blood sugar testing and normal levels of lipid profile and sugar.The . Diabetic patients, hypertensive patients, Patients who are on antiplatelet for any reason. . Patients who received steroid in the last month prior to including in the study and Patients with features of peripheral vascular disease (weak or absent Peripheral pulsation) were excluded from the study .

A control group including a twenty healthy control from hospital staff, 13 female, 7 male were included in the study, age was ranged between 23 and 45 . History was taken from patient according to a structural questionnaire and physical Examination was done according to a structural questionnaire ; Each patient was sending for IFAT test for brucellosis, vasculitic screen, MRI - MRV was done using 3 Tessla, Phillips model Achieva, 2012 and interpreted by a radiologist in Neurosciences Hospital, a special emphasis was considered for thrombosis in superficial and deep veins as the following:-

a. The clot acutely is isointense on T1 and hypointense on T2 (this can mimic a flow void), with subacute clot becoming hyperintense on $\mathrm{T} 1$.

b. MRV will demonstrate a lack of flow. 2D Time of Flight (TOF) venography.

Table [1] age distribution
C. Contrast MR Venography has more sensitive in detecting dural venous sinus thrombosis than TOF venography.

Statistical Analysis was done By using (IBM SPSS v.20) which is a software package used for statistical analysis in addition to usage (Microsoft excel 2010) for data sheet collection, the data of all cases were entered and analyzed; descriptive and analytic statistics were performed using an appropriate statistical configuration. All data were presented as tables, graphs or paragraph and in all statistical procedures and tests $P$-value set at $\leq 0.05$ to be considered as significant

Results :Table [1] shows age distribution of patients with MS, $44 \%$ of patients were between $31-40$ year old and $30 \%$ of patients were between $21-30$ and others age groups were seen in the table 3.1. All the patients with positive MRV not belongs to smoker or alcohol groups as seen in the table 3 . Figure one showed a $82 \%$ of MS is relapsing /remitting, $12 \%$ is secondary progressive, $4 \%$ progressive/relapsing and $2 \%$ primary progressive as seen in the figure. Table(4) shows $42 \%$ of MS cases have an EDSS between 4-5, and 38\% of cases have 3-4 EDSS. Figure 2 showed a $64 \%$ of MS presented with numbness, $48 \%$ is presented with walking difficulty, $40 \%$ vision problem and fatigue.Figure 3 showed a $8 \%$ of MS cases have MRV finding as cortical venous thrombosis. Figure 4 shows $76 \%$ of MS patients are treated with betaferon, $12 \%$ are treated with avonix and $12 \%$ are treated with Rebif

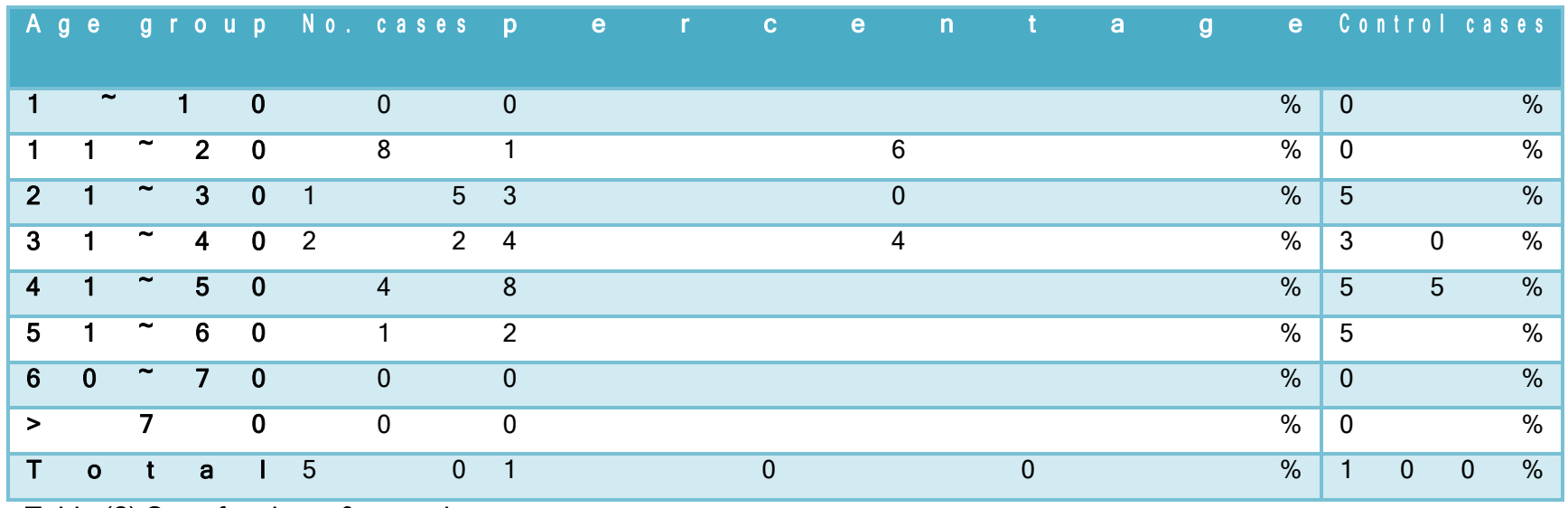

Table (2) Sex of patients \& control group

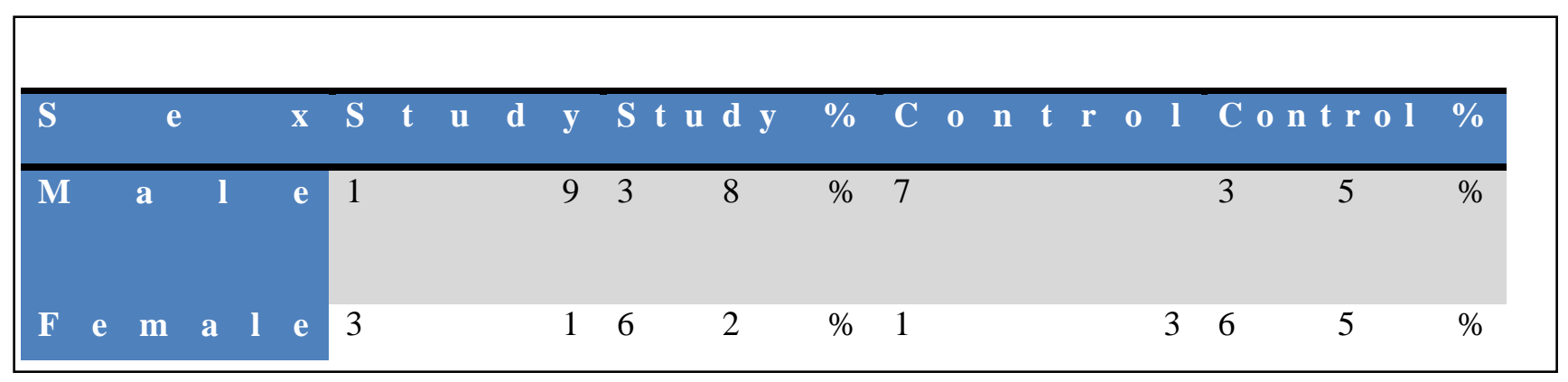


Table (3) Social history

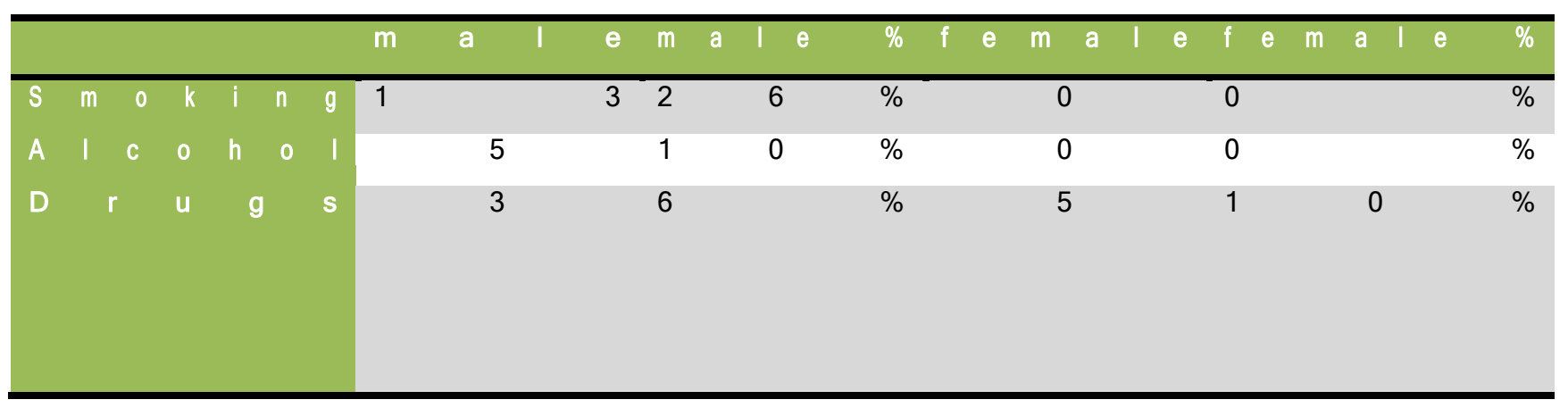

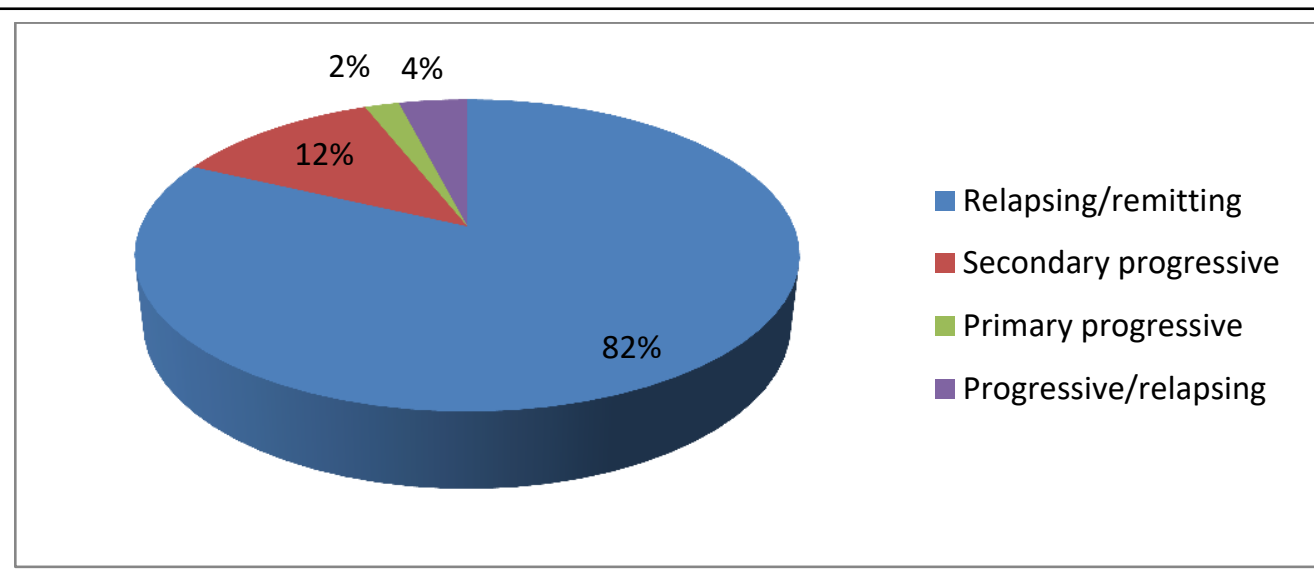

Figure (1) Types of MS

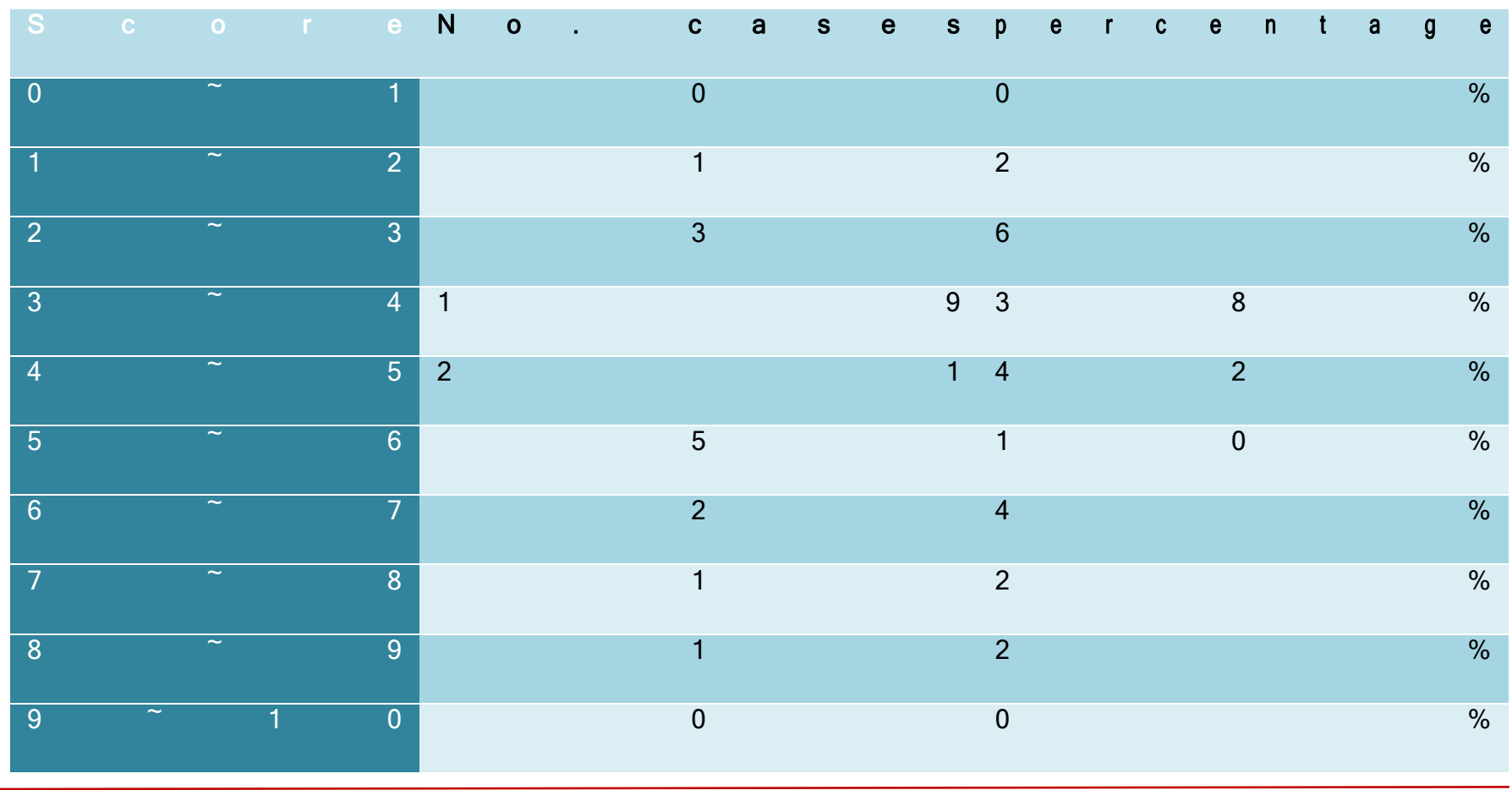



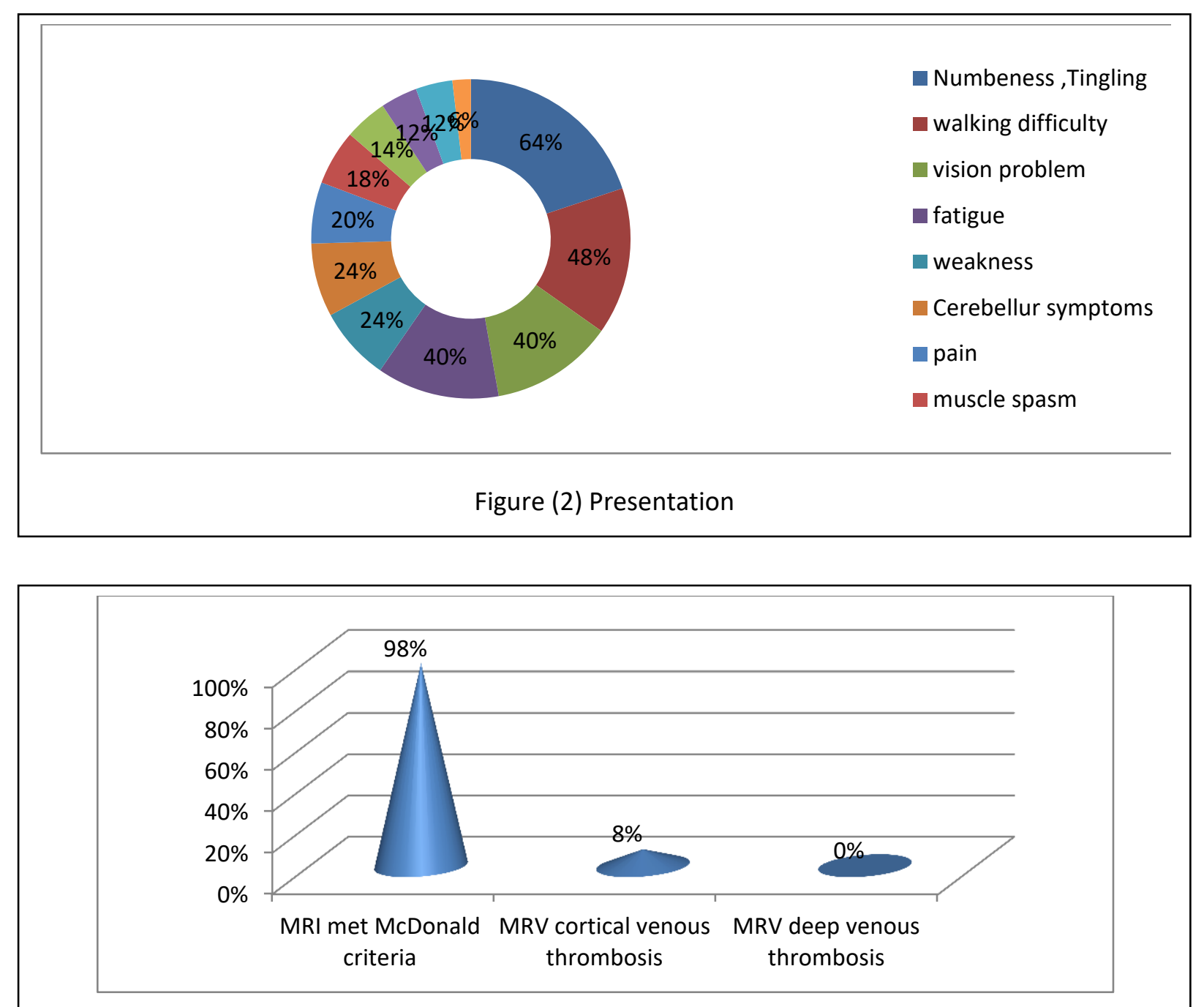

Figure (3) MRI Finding in MS

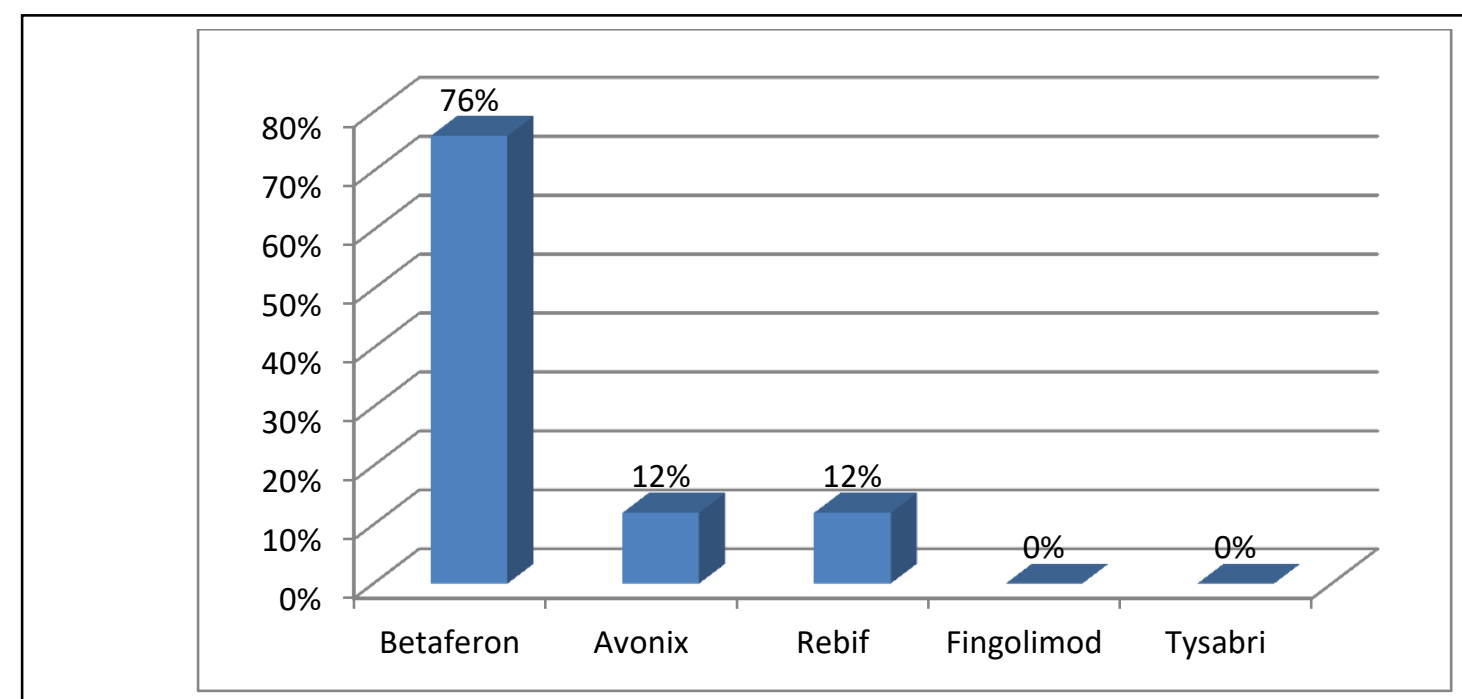

Figure (4) Treatment used in study cases 
Discussion :Thrombotic processes were reported in multiple sclerosis, but they are under estimation in clinical practice. This case- control study was aimed at evaluated the presence of cerebral venous insufficiency in multiple sclerosis patients. The present study argues against the positive relationship between the cerebral venous insufficiency and multiple sclerosis risk, and observed a weak association. The researcher in this study found that all the cases were between the age of 10-60 year old, and more than two third was between 20-40 year old, this goes with natural age distribution in multiple sclerosis and go with Daniele .I et al study(8). Regarding the gender distribution, the study show that two third of the cases was female and the rest was male, which agree with Lanzillo. $R$ et al study (9). There was no familiar basis for multiple sclerosis in the present study, which was disagree with Kalamin.B et al who revealed $1 \%$ of cases was familial and this disagreement may be due to small sample size of the present thesis (10). The current study found that most of the cases were a relapsing remitting one while only 6 cases (12\%) was a secondary progressive type, it doesn't go with Duddy. $\mathrm{M}$ et al study which had $30 \%$ of the cases was a secondary progressive one, this can be explained by short time period of the present study and we may need to fellow the patient for a longer time to detect the cases that transform from the RRMS to SPMS (11). The researcher found that the EDSS was between (3-5) in $80 \%$ of the studying cases, it agreed with Mike $P$ et al study (12). The present study had 4 patients (8\%) with cortical vein thrombosis documented by MRV of brain while 46 patients (92\%) show no evidence of thrombosis by MRV , no significant relationship between cerebrospinal venous insufficiency and multiple sclerosis was observed in the present study, in one hand it agrees with the result of Zamboni $P$ and colleagues study (13) which mentioned the presence of cortical venous insufficiency in multiple sclerosis patient, and in the other hand the result of the current study was statistically not significant ( $p$ value 0.25 ) which was not the same of Zamboni $\mathrm{P}$ and colleagues study (13) which showed a highly significant $p$ value, the agreement may be explained by the current evolving theory of venous basis for the multiple sclerosis pathogenesis which was published by Alberta health service (14); and show that constant drainage of inflammatory proteins through the major veins of the brain can encountered for the venous changes reported in some patients with multiple sclerosis, while the disagreement might be explained by many factors, all the published studies are heterogeneous in sample size ,blinding procedures, characteristics of control groups and the types of imaging techniques was used, this is highly suggested that the association is not so common to the point that might change the well-known pathogenesis of the multiple sclerosis. The researcher of this study found that only multiple sclerosis patients and not controls met the criteria for abnormal cerebral venous flow, since 4 out of 50 patients had cortical vein thrombosis while none of the 20 controls show such finding, this is totally go with Omar $\mathrm{K}$ et al study(15). Both compared studies show none significant $p$ value, again it can reflect the presence of venous insufficiency in some multiple sclerosis patients but the prevalence not significantly different across groups. The conducted thesis try to find if there was any correlation between cortical vein thrombosis and the disease modifying therapy was taking by the four patients who had cortical vein thrombosis, and it found 3 of the 4 patients was on Betaferon while one patient on Avonex, the result was statistically not significant since the $p$ value was 0.6 for the Betaferon, that finding agree with Fact Med analysis covering adverse side effect reports of BETAFERON (16).which found that 3 patients reported venous thrombosis and claimed that the thrombosis is only a reported side effect rather that correlated one. No association between cerebrospinal venous insufficiency status and multiple sclerosis features was observed in our study. In particular, cerebrospinal venous insufficiency-positive and cerebrospinal venous insufficiency-negative multiple sclerosis did not differ in disease course (progressive versus non-progressive multiple sclerosis) and EDSS score. Similar result was also reported by some author (17), but not by other. Moreover our result do not support the pathophysiological relevance of venous insufficiency, in addition, it is unlikely that anomalous venous system is directly associated with multiple sclerosis symptom and the disease course or even the course of multiple sclerosis even in patients with real pathological cerebral venous thrombosis since higher incidence of multiple sclerosis had not been reported. The researcher faced many limitations regarding obtaining of MRI-MRV of brain for many uncooperative patients which leads to small size of the sample and small size of control, another limitation was the short duration of collection of data which lasted for 8 months only. The paucity of similar studies to compare the collected data was another limitation.

In conclusion MRV findings suggestive of venous insufficiency was reported in four patients out of 50 of MS patients in the present study and it was not reported in any patient of the control group. There is no significant statistical differences between the MS group and control group regarding the MRV findings. These results were not supportive for the theory of vascular etiology of MS. References

1. Flemming K. , Lyell Jones . Pathology and Spectrum of Central Nervous System Inflammatory Demyelinating Diseases in Mayo Clinic Neurology Board Review (1 Pap/Psc Edition). Mayo Clinic Scientific Press 
2. Compston A, Coles A (October 2008). "Multiple sclerosis". Lancet $372 \quad$ (9648): 1502-17. doi:10.1016/S0140-6736(08)61620-7. PMID 18970977

3. Compston A, Coles A (April 2002). "Multiple sclerosis".Lancet 359(9313): $1221-31$. doi:10.1016/S0140-6736(02)08220-X. PMID 11955556

4. Murray ED, Buttner EA, Price BH ."Depression and Psychosis in Neurological Practice". In Daroff R, Fenichel G, Jankovic J, Mazziotta J. Bradley's neurology in clinical practice. (6th ed.). Philadelphia, PA: Elsevier/Saunders.

5. Zamboni P, Galeotti R, Menegatti E, et al. Chronic cerebrospinal venous insufficiency in patients with multiple sclerosis. J NeurolNeurosurg Psychiatry. 2009 Apr. 80(4):392-9.

6. Zivadinov R, Schirda C, Dwyer MG, et al. Chronic cerebrospinal venous insufficiency and iron deposition on susceptibility-weighted imaging in patients with multiple sclerosis: a pilot case-control study. IntAngiol. 2010 Apr. 29(2):158-75.

7. Study To Evaluate Treating Chronic Cerebrospinal Venous Insufficiency (CCSVI) in Multiple Sclerosis Patients http://clinicaltrials.gov/ct2/show/NCT01089686.

8. Imperiale D, et al. Chronic cerebrospinal venous insufficiency in multiple sclerosis: A sonographer-blinded case-control study. ClinNeurolNeurosurg ,August 2013Volume 115, Issue 8, Pages 1394-1398

9. Lanzillo et al.: Chronic cerebrospinal venous insufficiency in multiple sclerosis: a highly prevalent agedependent phenomenon. BMC Neurology 2013 13:20. 10.kalman B. et al., Familial multiple sclerosis and other inherited disorders of the white matter .Neurologist. 2004 Jul;10(4):201-15 .

11. Duddy M, Haghikia A, Cocco E et al. Managing MS in a changing treatment landscape. Journal of Neurology 2011; 258: 728-739.

12. Mike P. Wattjes et al. ,No association of abnormal cranial venous drainage with multiple sclerosis: An MR venography and flow-quantification study ,J neurolneurosurg psychiatry. Published Online: 09 October 2013 DOI: http://dx.doi.org/10.1016/S0140 6736(13)61912-1

13. Zamboni $P$, Galeotti R, Menegatti E, et al. A prospective open-label study of endovascular treatment of chronic cerebrospinal venous insufficiency. J Vasc Surg 2009;50:1348-1358

14. Alberta Health Services Information Sheet ,Multiple Sclerosis (MS) and "Chronic Cerebrospinal Venous Insufficiency" (CCSVI), August 6, 2010

15. Omar Khan, et al.: Chronic Cerebrospinal Venous Insufficiency and Multiple Sclerosis ,ANN NEUROL 2010;67:286-290.

16. Electronic published article :Study of possible correlation between MULTIPLE MYELOMA and BETAFERON (INTERFERON BETA - 1B) INJECTIO, 250UG[http://www.factmed.com/studyBETAFERON\%20 (INTERFERON\%20BETA\%20\%201B)\%20INJECTIO,\% 20250UG-causing-MULTIPLE\%20MYELOMA.php]

17. Centorlze D, Floris R, Stefanini M, Rossi S, Fabiano $S$, Castelli M. et a1. Proposed chronic cerebrospinal venous insufficiency criteria do not predict multiple sclerosis risk or severity. Annals of Neurology 2011;70:51-8. 